\title{
Tracking Urban Mobility and Occupancy under Social Distancing Policy
}

\author{
WENDY JU, SHARON YAVO-AYALON, and ILAN MANDEL, Jacobs Technion-Cornell Institute, \\ Cornell Tech \\ FEDERICO SALDARINI, Independent \\ NATALIE FRIEDMAN, Cornell Tech, United States \\ SRINATH SIBI, Stanford University, United States \\ J. D. ZAMFIRESCU-PEREIRA, University of California, Berkeley, United States \\ JORGE ORTIZ, Rutgers University, United States
}

\begin{abstract}
The effectiveness of social distancing as a disease-slowing measure is dependent on the degree of compliance that individuals demonstrate to such orders. In this ongoing research, we study outdoor pedestrian activity in New York City, specifically using (a) video streams gathered from public traffic cameras (b) dashcam footage from vehicles driving through the city, and (c) mobile phone geo-location data volunteered by local citizens. This project seeks to form a multi-scale map of urban mobility and space occupancy under social distancing policy. The data collected will enable researchers to infer the activities, contexts, origins, and destinations of the people in public spaces. This information can reveal where and, in turn, why stayat-home orders are and are not being followed. As a work in progress, it is yet too early for detailed findings on this project. However, we report here on several unanticipated factors that have already influenced the course of the project, among them: the death of George Floyd and subsequent protests, data collection challenges, changes in the weather, and the unexpected nature of the progression of COVID-19.
\end{abstract}

CCS Concepts: • Human-centered computing $\rightarrow$ Empirical studies in HCI; Geographic visualization; • Applied computing $\rightarrow$ Sociology;

Additional Key Words and Phrases: COVID-19, social distancing, urban mobility, New York city, geographic visualization

\section{ACM Reference format:}

Wendy Ju, Sharon Yavo-Ayalon, Ilan Mandel, Federico Saldarini, Natalie Friedman, Srinath Sibi, J. D. Zamfirescu-Pereira, and Jorge Ortiz. 2020. Tracking Urban Mobility and Occupancy under Social Distancing Policy. Digit. Gov.: Res. Pract. 1, 4, Article 32 (October 2020), 12 pages.

https://doi.org/10.1145/3417991

\footnotetext{
This work is supported by a grant from the National Science Foundation, IIS-2028009: RAPID: Tracking Urban Mobility and Occupancy under Social Distancing Policy.

Authors' addresses: W. Ju, S. Yavo-Ayalon, and I. Mandel, Jacobs Technion-Cornell Institute, Cornell Tech, 2 West Loop Rd, New York, NY, 10044; emails: \{wendyju, sharon.ayalon, im334\}@cornell.edu; F. Saldarini, Independent; email: fede.saldarini@gmail.org; N. Friedman, Cornell Tech, New York; email: nvf4@cornell.edu; S. Sibi, Stanford University, Stanford; email: sibisrinath@gmail.com; J. D. ZamfirescuPereira, University of California, Berkeley, Berkeley; email: jdz32@cornell.edu; J. Ortiz, Rutgers University, New Brunswick; email: jorge.ortiz@rutgers.edu.

Permission to make digital or hard copies of part or all of this work for personal or classroom use is granted without fee provided that copies are not made or distributed for profit or commercial advantage and that copies bear this notice and the full citation on the first page. Copyrights for third-party components of this work must be honored. For all other uses, contact the owner/author(s). (c) 2020 Copyright held by the owner/author(s)

2639-0175/2020/10-ART32

https://doi.org/10.1145/3417991
}

Digital Government: Research and Practice, Vol. 1, No. 4, Article 32. Publication date: October 2020. 


\section{INTRODUCTION}

On March 20th, 2020, New York Governor Andrew M. Cuomo issued a "stay at home" order to slow the spread of COVID-19. He instructed the majority of the public, excluding those working in essential occupations, not to go physically to work, nor to gather for parties, celebrations, or social events. This policy of social distancing has been established to reduce transmission and spread of infectious diseases such as pandemic influenza. Empirical case studies affirm predictions of mathematical models that indicate that even mild behavior changes can have a dramatic effect in slowing an epidemic [45]. However, the effectiveness of social distancing as a diseaseslowing measure is dependent on the degree of compliance that individuals take to such orders.

In this article, we describe an ongoing research project that studies outdoor pedestrian activity in New York City. To create a holistic view on the city, we collect data in three different scales: (a) The City scale is gathered through video streams gathered from public traffic cameras, (b) the Public scale is gathered through video footage taken from instrumented vehicles driving through city, and (c) the Individual scale is gathered through mobile phone location data volunteered by local citizens. These multi-scale data will be used to form a map of urban mobility and space occupancy under social distancing policy.

\section{BACKGROUND}

In 1854, amid an outbreak of cholera in London's Soho neighborhood, physician John Snow created a map of the cholera deaths that occurred at different households [65]. Snow's map illustrated that deaths were clustered around the Broad Street pump, convincingly enough that the local council removed the handle of the pump to discourage its use [34]. This story is often told to show how data can be collected and visualized to inform public policy to improve public health [57]. This project similarly aims to collect data to inform how people's movements and activities in the city respond to information and affect public health outcomes in the COVID-19 outbreak.

\subsection{Mobility and Viral Transmission within Cities}

Viral outbreaks are both natural and anthropogenic-their spread is directly tied to people's activity and movement. Wilson and Chen noted that SARS-CoV-2, the virus that causes COVID-19, spread most quickly and proportionally to the cities with the greatest passenger volume from the Wuhan International Airport [75]. Studies and models of viral transmission often focus on city-scale transmission, examining factors such as air travel or vehicular commute on the spread of viral disease $[8,50]$.

The question of how to contain spatial spread of infectious disease to prevent the overloading of medical capacity, however, speaks to the need to understand how to contain viral spread within cities and neighborhoods [11, 66, 67]. Traditional empirical studies of disease outbreaks are based on contact tracing (for example, References [7, 12, 14, 74, 77]); these methods break down when the infectious disease becomes more prevalent. The application of urban mobility tracking and urban space occupancy can help understand what activities and agents promote and prevent transmission of disease at a broader scale.

\subsection{Social Distancing Efficacy}

The observation of people's movement can help us to understand the efficacy of social distancing measures [73]. Research studying the effects of social distancing design has focused on the effect of high-level interventions such as closing schools and work locations or shutting down large conferences or social events that have the potential to infect many people who then disperse the virus geographically [4,30,55]. Studies of social distancing have been largely premised on physical models of droplet transmission and small-scale analysis of outbreak growth. From the 1930s to the early 2000s the social distancing guidelines suggested people stay three feet apart. This recommendation was based on work by W. F. Wells studying the physics of droplet transmission though the air [71]. The three-foot guideline was only revised to six feet after an analysis of disease transmission

Digital Government: Research and Practice, Vol. 1, No. 4, Article 32. Publication date: October 2020. 
on a flight during the SARS epidemic of 2003 [53]. Recent scholarship of transmission of COVID-19 specifically has further complicated these general guidelines [17, 37, 62]. The data being collected in this study contribute to the understanding of transmission at a scale between the individual case studies of transmission and larger scale epidemiological models of transmission.

Epidemiological models of disease outbreaks suggest that individual behaviors and compliance with public health directives can strongly influence how quickly a disease spreads [22]. In Hong Kong, for example, the prevalence of mask wearing and the avoidance of crowds helped to reduce the spread of COVID-19 through a densely populated city [18]. Observations of differences in infection rates in neighborhoods exhibiting different individual behaviors-for instance, whether people gather in large or small groups or whether they wear face masks-can help to tease apart the effects of large-scale social distancing measures from the effects of individual compliance and behavior.

Collection of data on urban mobility over the course of a disease outbreak also makes it possible to examine the role of information and policy on social behavior, and, in turn, on health outcomes. These data make it possible to see the differential effects of changes in policy. During the flu epidemic of 191819, for example, extensive infection control measures were imposed in Sydney, Australia: Theaters were closed, masks were made compulsory, meetings and church services were prohibited. Once these measures were lifted, however, the public resumed normal behavior, and this caused another wave of sicknesses and deaths that led to the resumption of control measures [15, 47]. Longitudinal tracking can also be used to map the effectiveness of public health information campaigns. Knowledge of epidemic outbreaks can cause people to voluntarily perform social distancing [27] but also to flee epidemic centers and thereby spread infectious diseases [48].

\subsection{Individual Factors in Compliance}

Compliance is a critical contributor to the effectiveness of social distancing policy, yet enforcement of social distancing measures is difficult. Many epidemiologists argue that the socio-economic status, role, or connectivity of individual actors in larger social networks needs to be accounted for in modelling contagion [11, 25, 40, 67]. Research based on surveys and focus groups about social distancing and influenza indicates that young people [63], men [24], people with job insecurity or no sick leave [13], and people who do not trust government [10] are less likely to comply with social distancing measures.

Understanding the differential incentives and motivating factors [56], as well as differences in the way that information is conveyed to different social actors [25], can be important to understanding how to design social policies or information campaigns to specifically address the needs, concerns, and motivations of different people in the larger public. By using collected data to infer characteristics of people moving through public spaces, such as age, gender presentation, role, and activities, we can better address individual factors in compliance. We feel that, for privacy reasons, our approach is preferable to gaining similar knowledge through tracking of actual individuals.

\subsection{Technologies for Tracking Urban Mobility and Occupancy}

Technology enables us to collect information about who is moving through the city, for what purpose, and where and why people might be clustering.

Because mobile phones often collect location data by default, they can be used to track people's trajectories, transportation modes, dynamic contacts, and disease dynamics in an urban environment [32, 69, 72, 76]. Tizzoni et al. found that mobile phone use captures $87 \%$ of empirical travel data, and that it can be a good proxy for modeling epidemics [68]. To be useful in tracking infectious disease, this data needs to be linked to demographic, social interaction, and disease status factors [72]. Public web cameras are also being used to track occupancy of urban spaces and movement patterns of individuals and groups [35, 60, 64], and to estimate pedestrian volume $[43,54]$. Although these cameras are sparsely distributed throughout urban spaces, vehicle-mounted cameras driving through urban and suburban areas can also be used to track road-side activities [38]. 
Computer vision can be used to infer individual factors, behaviors, and activities in video. Recognition techniques have been developed to estimate the gender and age of pedestrians based on gait [44, 46]; similar methods might be used to infer activity or role through identification of equipment or uniforms that people are holding or wearing. Prior research uses video to identify whether people are congregating in large or small groups [28, 49] or moving in social groups within larger crowds [39]. Techniques such as pose estimation can be leveraged for analysis of people's physical and spatial arrangement in video data [16]. Socio-economic attributes of a neighborhood including income, race, housing prices, and voting patterns have been successfully inferred from street view image data collected from roving vehicles [6, 29, 42]. Data collected from public cameras and mobile phones can be used to better understand how people are moving through the city during the COVID-19 pandemic and New York City's social distancing orders, to identify social behaviors and their relation to policy directives and health outcomes.

\section{METHODOLOGY}

Building upon prior research collecting empirical data about pedestrian and other road-user behavior and interactions [20,41,58], as well as work gathering large datasets of on-road data for machine learning about human behavior [9, 61], we are collecting data about urban mobility and occupancy of pubic space in New York City under social distancing policy. Our data collection methodology aims to create a multidimensional perspective of the city, taking into consideration different scales and changes over time. The three sources of data collection represent different scales of observing the city:

(1) The top-down City scale is gathered through video streams captured from public traffic cameras found throughout New York.

(2) The street level Public scale is gathered through video footage taken from instrumented vehicles driving through city streets.

(3) The bottom-up Individual scale is gathered through mobile phone location data volunteered by local citizens.

\section{WORK IN PROGRESS}

The research activity for this project commenced in the middle of April 2020, about a month after the "Stay at Home" order was issued in New York State. To build the multi-scale perspective described above, we assembled an interdisciplinary team with researchers from information sciences, urban planning and design, human-robot interaction, and computer science. We have consulted an epidemiologist and urban researchers to make sure our approach to data collection will be as relevant as possible to these other disciplines. We have made early effort to publicly communicate our activity and goals. This is primarily centered on the project website, which describes the project objectives in lay terms, including a short video. The website, https://www.socialdistancing. tech.cornell.edu/, helps us recruit volunteers and participants through social networks for data collection, and is also a resource for potential partners as we ramp up our efforts. We intend to use the site to report on ongoing progress and initial results as well. At the end of the process, it will be used to disseminate the research results to a wider audience beyond academia.

\subsection{The City-Top-down Public Webcam Data Collection}

The NYC Department of Transportation (DOT) maintains a list of approximately 650 publicly accessible webcams for real-time traffic estimation [2]. These cameras are not evenly distributed, rather they focus on major crosswalks, intersections, and roadways. The cameras update approximately once per second, and we download images approximately once every 90 seconds to 10 minutes, depending on server response times. The fixed nature of these cameras provides regular change in pedestrian traffic within each camera's view. Unlike aggregated activity reports provided by large tech companies [5,33], these cameras capture the enacted physical

Digital Government: Research and Practice, Vol. 1, No. 4, Article 32. Publication date: October 2020. 


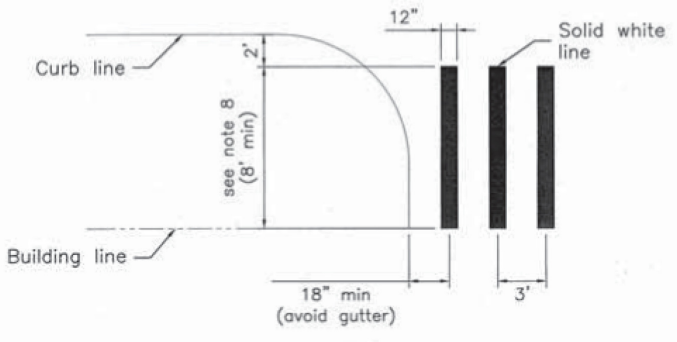

(a) Crosswalk marking dimensions are provided by the NYC Department of Transportation's technical drawings for Typical Pavement Markings [52]

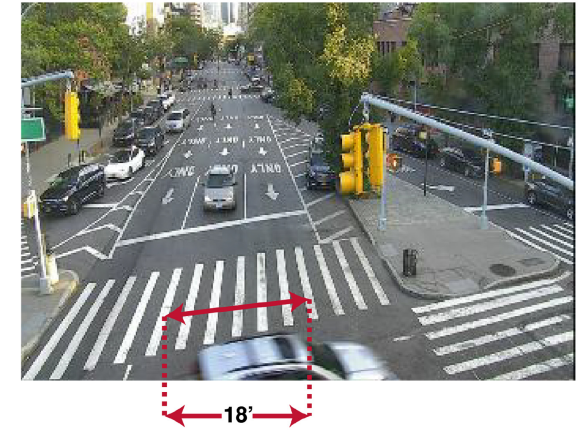

(b) Distances can be approximated via inverse geometric transformations of known shapes and patterns

Fig. 1. For each camera, we can use standard street markings to determine reasonable estimations of distance with no knowledge of camera characteristics.

behaviors of social distancing. Leveraging standardized artifacts of the urban environment, we can accurately estimate distances in many of these camera video feeds as can be seen in Figure 1.

These data give us comprehensive information about the number of people in the same location over time. Applying machine learning algorithms to the images collected, we have generated an empirical count of the number of people at each site. We are also exploring automated methods to determine whether the people visible in these images are wearing a mask. These images also enable comparative analysis of occupancy over different times of day (morning, afternoon, or evening, and potentially overnight), over weekdays and weekends, and different local weather conditions (for example, a rainy day or a sunny day).

The primary limitation of this dataset is that the existing traffic web cameras' installation locations do not necessarily match the regions of greatest interest with respect to social distancing behavior. There are, for example, far fewer cameras in the Bronx or Queens, where there were major early outbreaks of COVID-19, than in Manhattan and Brooklyn. Nevertheless, this dataset helps us to calibrate the data collected at other scales so we can better estimate the net pedestrian traffic in places where top-down public data are not available.

\subsection{The Public-Street-level Data Collection}

To build the street-level perspective, we are collecting on-road data from dashboard cameras ("dashcams") of vehicles driving throughout NYC. The types of data we intend to derive from this strata of data are shown in Figure 3. At first, we performed our own data collection, borrowing methodology we developed through previous research for on-road behavior $[9,61]$. This was done for two weeks to develop a working protocol and to better understand what data can be reasonably captured. After initial analysis of this early data, we have narrowed focus on the important parameters to visualize and the geographical areas that we want to cover.

To scale up data collection efforts beyond what our research team can personally collect with our single research vehicle, we developed a protocol for using Mapillary's [1] mobile phone app to capture specific routes in each borough several times per week (see Figure 2). We initially recruited drivers through TaskRabbit [3], and each driver was provided with equipment to collect public road-side imagery to the Mapillary database. We assigned one driver for each borough, prescribed driving routes, and monitored their submitted image streams.

More recently, we have also established a data exchange relationship with Nexar, a company that collects data from connected dashcams deployed in Uber, Lyft, and city taxis to improve road safety. We have licensed access to Nexar's database of NYC street-level images for the period six months prior to the "Stay at Home" order and three months after. This dataset enables us to form a more comprehensive map of street occupancy over time. 


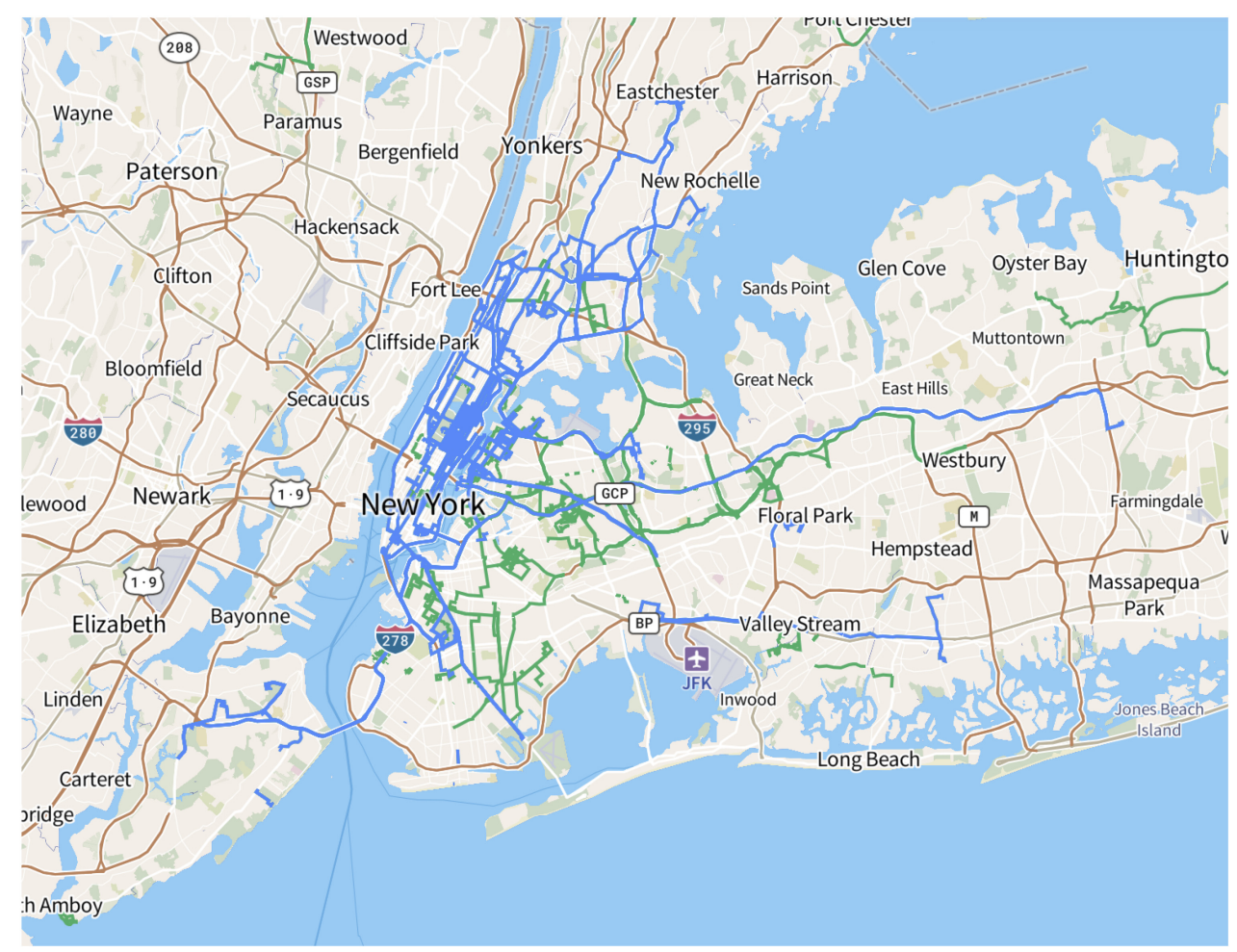

Fig. 2. All Mapillary public data collected since March 1, 2020. FAR Lab’s data are in blue.
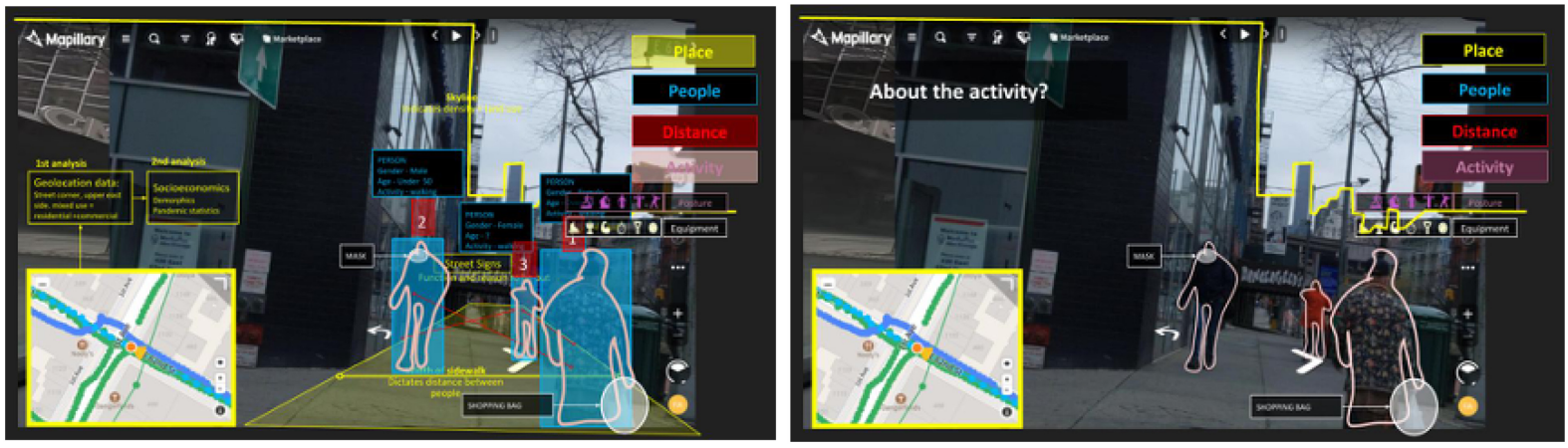

Fig. 3. Computational analysis to be performed on the Public-scale captured data.

Our Public-scale dataset gives us more nuanced understanding of the street and its small-scale human interactions. These data will give us a deeper understating of how people are adapting their behavior and the urban space around them to current social distancing policy. Using this granular street-level imagery, our analysis aims to track:

Changes in the way people are using the built environment. We are analyzing the spatial dimensions of the street (width of sidewalk, location of garbage bins, presence of an essential institution) in relation to the people in them to find correlation or divergence. We are also observing the skyline of the street to 
see if there is correlation between street section and pandemic statistics-for example, does an area with a wider, more open street section have less infections?

Changes in the way people are socializing with each other. Initial observations indicate a new "sidewalk ballet" [36] of people moving slightly away from one another, using objects in the street to create a divide between groups, or crossing the street to avoid contact.

Changes in people's activities. By training computer vision algorithms to detect objects people are carrying with them, such as a shopping bag, a dog leash, or a tennis racquet, we can infer the different activities people are pursuing under social distancing.

\subsection{The Individual-Bottom-up Mobile Phone Data Collection}

To build the individual person perspective, we are zooming in to one-person routines and routes throughout the social distancing policy duration. To reach as many volunteers as possible, we created a website that includes the main objectives of the project. Through advertising this website via social network platforms, we are recruiting volunteers from the greater New York City area to share their geotracking data collected from Google Takeout and Apple Location History [21]. To further deepen our understanding on the individual perspective, we are supplementing this data collection with a questionnaire that includes information about employment, gender, health status, and self-reported compliance with social distancing measures. This information is collected with consent and does not include personally identifiable information. We elicit information about the general location of each participant's work and home and perform obfuscation of location data to avoid revealing participants' identities. Collecting the routes of specific people over time will enable deeper understanding of new routines, patterns of movements, and activities of different stakeholders. The questionnaire data attached to the routes will help us perform statistical calibration of compliance with social distancing according to age, gender, health status, employment status, and so forth.

\section{ETHICAL CONSIDERATIONS FOR DATA COLLECTION AND SHARING}

The proposed research raises several safety and ethics considerations, which we address here.

Data collection and surveillance. This research involves the collection of data that can be personally identifiable. Although we believe that our work will have immediate and positive consequences for public health, we are mindful that the methods that we are using to contain contagion can also be used by authoritarian governments to monitor social assemblies and protests [70]. To this end, consideration of how to manage and mitigate risks of misuse are an active part of this research.

Contextual integrity. The specific issue of privacy depends on ethical norms surrounding information capture and use [51]. People's expectations of privacy during disasters differs from non-emergency situations [59], so we will also employ differential privacy measures [23] to make sure data are shared.

Risks to participants, the public, and others. We will develop a risk-mitigation checklist to minimize potential for harm from vehicles involved in our data collection efforts. It is possible that this dataset might also capture criminal activity or civil infractions; since the purpose of this data collection is to monitor human activity for public health reasons, we aim to use the data only for these purposes to the fullest extent allowable by law and with protections appropriate to the context of use.

\section{EMERGING ISSUES AND OUTCOMES}

This project is in its initial stages, so it is as yet too early for detailed discussion. Already there were a large number of changes and circumstances affecting our research that we could not have anticipated. Here are some of the issues that have emerged:

Digital Government: Research and Practice, Vol. 1, No. 4, Article 32. Publication date: October 2020. 
Responses to changes in weather. The "stay at home" order was issued on March 20, with NYC winter conditions forcing people to spend most of their time indoors. However, as the weather warmed, more people headed outdoors to parks [31]. Whether or not these outdoor spaces are healthful or harmful is a matter of debate, the type that our research is intended to help address, so these spaces are now important locations to also instrument and observe. To adapt our data collection to changing conditions, we are working on a strategy to recruit more volunteers to collect data in parks. Our goal here is to fill the gaps between places that are accessible by cars by including those places that are only open to pedestrians.

The killing of George Floyd and subsequent protests. The public response to events in Minnesota and longstanding inequities in American society have led to massive public protests worldwide and in New York City. Additional public activity on the street level has created different mobility patterns unrelated and in conflict with social distancing policy. To analyze this period, we plan to take into consideration those activities and focus on social distancing compliance within a crowded area. This information could be of great importance as a comparative dataset. In addition, the protest activity made it harder to collect some of our data. For example, the New York City Department of Transportation's public web cameras popularity as a source of information for activists tracking police activity [26] has affected the rate of camera data supplied at key intersections.

The progression of COVID-19 has been stemmed in New York City and New York State, but has risen dramatically elsewhere, making travel into New York a key concern [19]. We anticipate that these will be aspects of the research that we will be focusing on when we subsequently summarize the research at a later date.

Changes in social distancing policies. The media coverage and governmental campaigns change constantly, and with them, public behavior. These impacts also will be an aspect to focus on in the analysis phase, to check for correlations between governmental campaigns and the way people behave on the street.

The reopening of NYC. As New York City begins its phased reopening, many unprecedented changes in the use of public spaces are occurring. Streets are being closed to vehicular movements to prioritize pedestrians. Restaurants, bars, and cafés are taking over sidewalk spaces to enable dining outside. Social distancing lines are forming on many streets as people wait for takeout or to enter grocery stores. All those changes are being documented in our multi-scale datasets.

The analysis of the collected data will be useful for evaluating the efficacy of various reopening strategies. Moreover, through this analysis, we can better understand how people are adapting their behaviors of daily life in urban environments. While we do not know what will come next, it is clear that the transformations of the city will be instrumental to future public health and social policy design. By anticipating and instrumenting these changes using various overlapping measures, we hope to help inform planning for this and future pandemics.

\section{ACKNOWLEDGMENTS}

Thanks to the New York City Department of Transportation and the Nexar Corporation for their assistance and support in our data collection efforts.

\section{REFERENCES}

[1] Mapillary. Retrieved from https://www.mapillary.com/.

[2] Real-Time Traffic Information. Retrieved from https://webcams.nyctmc.org/multiview2.php.

[3] Taskrabbit. Retrieved from http://taskrabbit.com.

[4] Marco Ajelli, Piero Poletti, Alessia Melegaro, and Stefano Merler. 2014. The role of different social contexts in shaping influenza transmission during the 2009 pandemic. Sci. Rep. 4 (2014), 7218. Retrieved from https://www.nature.com/articles/srep07218.

[5] Apple. 2020. COVID-19 Mobility Trends Reports. Retrieved from https://www.apple.com/covid19/mobility.

[6] Sean M. Arietta, Alexei A. Efros, Ravi Ramamoorthi, and Maneesh Agrawala. 2014. City forensics: Using visual elements to predict non-visual city attributes. IEEE Trans. Vis. Comput. Graph. 20, 12 (2014), 2624-2633.

[7] Mike Baker and Karen Weise. 2020. Coronavirus hits a nursing home and a retirement complex in Seattle area. New York Times (6 Mar. 2020). Retrieved from https:/www.nytimes.com/2020/03/06/us/coronavirus-washington-state.html.

Digital Government: Research and Practice, Vol. 1, No. 4, Article 32. Publication date: October 2020. 
[8] Duygu Balcan, Vittoria Colizza, Bruno Gonçalves, Hao Hu, José J. Ramasco, and Alessandro Vespignani. 2009. Multiscale mobility networks and the spatial spreading of infectious diseases. Proc. Nat. Acad. Sci. 106, 51 (2009), 21484-21489. Retrieved from https://www.pnas.org/content/106/51/21484.short.

[9] Sonia Baltodano, Jesus Garcia-Mancilla, and Wendy Ju. 2018. Eliciting driver stress using naturalistic driving scenarios on real roads. In Proceedings of the 10th International Conference on Automotive User Interfaces and Interactive Vehicular Applications. 298-309.

[10] Nancy M. Baum, Peter D. Jacobson, and Susan D. Goold. 2009. "Listen to the People": Public deliberation about social distancing measures in a pandemic. Amer. F. Bioeth. 9, 11 (2009), 4-14. Retrieved from https://www.ncbi.nlm.nih.gov/pubmed/19882444.

[11] Vitaly Belik, Theo Geisel, and Dirk Brockmann. 2011. Natural human mobility patterns and spatial spread of infectious diseases. Phys. Rev. X 1, 1 (2011), 011001. Retrieved from https://journals.aps.org/prx/abstract/10.1103/PhysRevX.1.011001\#fulltext.

[12] Qifang Bi, Yongsheng Wu, Shujiang Mei, Chenfei Ye, Xuan Zou, Zhen Zhang, Xiaojian Liu, Lan Wei, Shaun A. Truelove, Tong Zhang et al. 2020. Epidemiology and transmission of COVID-19 in Shenzhen China: Analysis of 391 cases and 1,286 of their close contacts. medRxiv. Retrieved from https://www.medrxiv.org/content/10.1101/2020.03.03.20028423v2.

[13] Kelly D. Blake, Robert J. Blendon, and Kasisomayajula Viswanath. 2010. Employment and compliance with pandemic influenza mitigation recommendations. Emerg. Infect. Dis. 16, 2 (2010), 212. Retrieved from https://www.ncbi.nlm.nih.gov/pmc/articles/PMC2958001/.

[14] Jing Cai, Wenjie Sun, Jianping Huang, Michelle Gamber, Jing Wu, and Guiqing He. 2020. Early release-indirect virus transmission in cluster of COVID-19 cases, Wenzhou, China, 2020. Emerg. Infect. Dis. 26, 6 (June 2020). Retrieved from https://wwwnc.cdc.gov/eid/ article/26/6/20-0412_article.

[15] Peter Caley, David J. Philp, and Kevin McCracken. 2008. Quantifying social distancing arising from pandemic influenza. F. Roy. Soc. Interf. 5, 23 (2008), 631-639. Retrieved from https://royalsocietypublishing.org/doi/full/10.1098/rsif.2007.1197?casa_token= BkfN5vnGLaIAAAAA\%3ANxmimR6sdbyYA5jAtHf-bFj-st2RtJIToD9cyXPiGAeQ0u_cYFHxvI4g4hWMkAr-7y-vqqstLlU0iImA.

[16] Zhe Cao, Gines Hidalgo, Tomas Simon, Shih-En Wei, and Yaser Sheikh. 2018. OpenPose: Realtime multi-person 2D pose estimation using Part Affinity Fields. arXiv preprint arXiv:1812.08008 (2018).

[17] Derek K. Chu, Elie A. Akl, Stephanie Duda, Karla Solo, Sally Yaacoub, Holger J. Schünemann, Amena El-harakeh, Antonio Bognanni, Tamara Lotfi, Mark Loeb et al. 2020. Physical distancing, face masks, and eye protection to prevent person-to-person transmission of SARS-CoV-2 and COVID-19: A systematic review and meta-analysis. Lancet 395, 10242 (7 June 2020) P1973-1987.

[18] Benjamin J. Cowling, Sheikh Taslim Ali, Tiffany W. Y. Ng, Tim K. Tsang, Julian C. M. Li, Min Whui Fong, Qiuyan Liao, Mike Y. W. Kwan, So Lun Lee, Susan S. Chiu et al. 2020. Impact assessment of non-pharmaceutical interventions against COVID-19 and influenza in Hong Kong: An observational study. medRxiv. Retrieved from https://www.medrxiv.org/content/10.1101/2020.03.12.20034660v1.

[19] Andrew Cuomo. 2020. Governor Cuomo Announces Individuals Traveling to New York from Eight Additional States Will Be Required to Quarantine for 14 Days. Retrieved from https://www.governor.ny.gov/news/governor-cuomo-announces-individuals-traveling-newyork-eight-additional-states-will-be.

[20] Rebecca Currano, So Yeon Park, Lawrence Domingo, Jesus Garcia-Mancilla, Pedro C. Santana-Mancilla, Victor M. Gonzalez, and Wendy Ju. 2018. ¡Vamos! Observations of pedestrian interactions with driverless cars in Mexico. In Proceedings of the 10th International Conference on Automotive User Interfaces and Interactive Vehicular Applications. 210-220.

[21] Andrew Dancy. 2020. How to Export \& View iPhone Location Data. Retrieved from https://reincubate.com/support/how-to/exportview-iphone-location-data/.

[22] S. Del Valle, H. Hethcote, J. M. Hyman, and C. Castillo-Chavez. 2005. Effects of behavioral changes in a smallpox attack model. Math. Biosci. 195, 2 (2005), 228-251. DOI : https://doi.org/10.1016/j.mbs.2005.03.006

[23] Cynthia Dwork. 2008. Differential privacy: A survey of results. In Proceedings of the International Conference on Theory and Applications of Models of Computation. Springer, 1-19. Retrieved from https://ink.springer.com/chapter/10.1007/978-3-540-79228-4_1.

[24] Keith Eastwood, David Durrheim, J. Lynn Francis, Edouard Tursan d'Espaignet, Sarah Duncan, Fakhrul Islam, and Rick Speare. 2009. Knowledge about pandemic influenza and compliance with containment measures among Australians. Bull. World Health Organiz. 87 (2009), 588-594. Retrieved from https://www.ncbi.nlm.nih.gov/pmc/articles/PMC2733278/.

[25] Abdulrahman M. El-Sayed, Peter Scarborough, Lars Seemann, and Sandro Galea. 2012. Social network analysis and agent-based modeling in social epidemiology. Epidem. Perspect. Innov. 9, 1 (2012), 1. Retrieved from https://link.springer.com/article/10.1186/1742-55739-1.

[26] Lorenzo Franceschi-Bicchierai. 2020. Activists Are Using Traffic Cameras to Track Police Brutality. Retrieved from https://www.vice. com/en_us/article/y3zp55/activists-are-using-traffic-cameras-to-track-police-brutality.

[27] Sebastian Funk, Erez Gilad, Chris Watkins, and Vincent A. A. Jansen. 2009. The spread of awareness and its impact on epidemic outbreaks. Proc. Nat. Acad. Sci. 106, 16 (2009), 6872-6877. Retrieved from https://www.pnas.org/content/106/16/6872.short.

[28] Weina Ge, Robert T. Collins, and Barry Ruback. 2009. Automatically detecting the small group structure of a crowd. In Proceedings of the Workshop on Applications of Computer Vision (WACV'09). IEEE, 1-8.

[29] Timnit Gebru, Jonathan Krause, Yilun Wang, Duyun Chen, Jia Deng, Erez Lieberman Aiden, and Li Fei-Fei. 2017. Using deep learning and Google Street View to estimate the demographic makeup of neighborhoods across the United States. Proc. Nat. Acad. Sci. 114, 50 (2017), 13108-13113. 
[30] Robert J. Glass, Laura M. Glass, Walter E. Beyeler, and H. Jason Min. 2006. Targeted social distancing designs for pandemic influenza. Emerg. Infect. Dis. 12, 11 (2006), 1671. Retrieved from https://www.ncbi.nlm.nih.gov/pmc/articles/PMC3372334/.

[31] Joseph Goldstein and Corey Kilgannon. 2020. Balmy Weekend Presents a Challenge: New Yorkers Rushing to Parks. New York Times (2 May 2020). Retrieved from https://www.nytimes.com/2020/05/02/nyregion/weather-parks-nyc-nj-coronavirus.html.

[32] Marta C. Gonzalez, Cesar A. Hidalgo, and Albert-Laszlo Barabasi. 2008. Understanding individual human mobility patterns. Nature 453, 7196 (2008), 779-782. Retrieved from https://www-nature-com.proxy.library.cornell.edu/articles/nature06958.

[33] Google. 2020. COVID-19 Community Mobility Reports. Retrieved from https:/www.google.com/covid19/mobility/.

[34] Sandra Hempel. 2007. The Strange Case of the Broad Street Pump: John Snow and the Mystery of Cholera. University of California Press.

[35] J. Aaron Hipp, Alicia Manteiga, Amanda Burgess, Abby Stylianou, and Robert Pless. 2016. Webcams, crowdsourcing, and enhanced crosswalks: Developing a novel method to analyze active transportation. Front. Pub. Health 4 (2016), 97. Retrieved from https://www. ncbi.nlm.nih.gov/pmc/articles/PMC4871890/.

[36] Jane Jacobs. 2016. The Death and Life of Great American Cities. Vintage.

[37] Mahesh Jayaweera, Hasini Perera, Buddhika Gunawardana, and Jagath Manatunge. 2020. Transmission of COVID-19 virus by droplets and aerosols: A critical review on the unresolved dichotomy. Environ. Res. 188 (Sept. 2020), 109819.

[38] Hirokatsu Kataoka, Yoshimitsu Aoki, Yutaka Satoh, Shoko Oikawa, and Yasuhiro Matsui. 2015. Fine-grained walking activity recognition via driving recorder dataset. In Proceedings of the IEEE 18th International Conference on Intelligent Transportation Systems. IEEE, 620-625. Retrieved from https://ieeexplore.ieee.org/abstract/document/7313199.

[39] Sultan Daud Khan, Giuseppe Vizzari, Stefania Bandini, and Saleh Basalamah. 2015. Detection of social groups in pedestrian crowds using computer vision. In Proceedings of the International Conference on Advanced Concepts for Intelligent Vision Systems. Springer, 249-260.

[40] Marek Laskowski, Bryan C. P. Demianyk, Julia Witt, Shamir N. Mukhi, Marcia R. Friesen, and Robert D. McLeod. 2011. Agent-based modeling of the spread of influenza-like illness in an emergency department: A simulation study. IEEE Trans. Inf. Technol. Biomed. 15, 6 (2011), 877-889. Retrieved from https://ieeexplore.ieee.org/abstract/document/5970118.

[41] Jamy Li, Rebecca Currano, David Sirkin, David Goedicke, Hamish Tennent, Aaron Levine, Vanessa Evers, and Wendy Ju. 2020. On-road and online studies to investigate beliefs and behaviors of Netherlands, US and Mexico pedestrians encountering hidden-driver vehicles. In Proceedings of the ACM/IEEE International Conference on Human-robot Interaction. 141-149.

[42] Xiaojiang Li, Chuanrong Zhang, Weidong Li, Yulia A. Kuzovkina, and Daniel Weiner. 2015. Who lives in greener neighborhoods? The distribution of street greenery and its association with residents' socioeconomic conditions in Hartford, Connecticut, USA. Urb. Forest. Urb. Green. 14, 4 (2015), 751-759.

[43] XiaoHang Liu and Julia Griswold. 2009. Pedestrian volume modeling: A case study of San Francisco. Yearb. Assoc. Pac. Coast Geogr. (2009), 164-181.

[44] Jiwen Lu and Yap-Peng Tan. 2010. Gait-based human age estimation. IEEE Trans. Inf. Forens. Secur. 5, 4 (2010), 761-770. Retrieved from https://ieeexplore.ieee.org/document/5557806.

[45] Benjamin F. Maier and Dirk Brockmann. 2020. Effective containment explains subexponential growth in recent confirmed COVID-19 cases in China. Science 368, 6492 (2020), 742-746.

[46] Yasushi Makihara, Hidetoshi Mannami, and Yasushi Yagi. 2010. Gait analysis of gender and age using a large-scale multi-view gait database. In Proceedings of the Asian Conference on Computer Vision. Springer, 440-451.

[47] Howard Markel, Harvey B. Lipman, J. Alexander Navarro, Alexandra Sloan, Joseph R. Michalsen, Alexandra Minna Stern, and Martin S. Cetron. 2007. Nonpharmaceutical interventions implemented by US cities during the 1918-1919 influenza pandemic. f. Amer. Med. Assoc. 298, 6 (2007), 644-654. Retrieved from https://jamanetwork.com/journals/jama/fullarticle/208354.

[48] Sandro Meloni, Nicola Perra, Alex Arenas, Sergio Gómez, Yamir Moreno, and Alessandro Vespignani. 2011. Modeling human mobility responses to the large-scale spreading of infectious diseases. Sci. Rep. 1 (2011), 62. Retrieved from https://www.nature.com/articles/ srep00062? page $=3$.

[49] Mehdi Moussaïd, Dirk Helbing, Simon Garnier, Anders Johansson, Maud Combe, and Guy Theraulaz. 2009. Experimental study of the behavioural mechanisms underlying self-organization in human crowds. Proc. Roy. Soc. B: Biolog. Sci. 276, 1668 (2009), $2755-2762$. Retrieved from https://royalsocietypublishing.org/doi/full/10.1098/rspb.2009.0405.

[50] Laura Nic Lochlainn, Sema Mandal, Rita de Sousa, Karthik Paranthaman, Rob van Binnendijk, Mary Ramsay, Susan Hahné, and Kevin E. Brown. 2016. A unique measles B3 cluster in the United Kingdom and the Netherlands linked to air travel and transit at a large international airport, February to April 2014. Eurosurveillance 21, 13 (2016). DOI : https://doi.org/10.2807/1560-7917.ES.2016.21.13.30177

[51] Helen Nissenbaum. 2004. Privacy as contextual integrity. Wash. Law Rev. 79 (2004), 119.

[52] NYC Department of Transportation. 2017. Crosswalks and Stop Bars. Retrieved from http://www.nyc.gov/html/dot/downloads/pdf/ nycdot_highwaydesign_typicalmarkings.pdf/.

[53] Sonja J. Olsen, Hsiao-Ling Chang, Terence Yung-Yan Cheung, Antony Fai-Yu Tang, Tamara L. Fisk, Steven Peng-Lim Ooi, Hung-Wei Kuo, Donald Dah-Shyong Jiang, Kow-Tong Chen, Jim Lando et al. 2003. Transmission of the severe acute respiratory syndrome on aircraft. New Eng. J. Med. 349, 25 (2003), 2416-2422.

Digital Government: Research and Practice, Vol. 1, No. 4, Article 32. Publication date: October 2020. 
[54] Anna Petrasova, J. Aaron Hipp, and Helena Mitasova. 2019. Visualization of pedestrian density dynamics using data extracted from public webcams. ISPRS Int. J. Geo-inf. 8, 12 (2019), 559. Retrieved from https://www.mdpi.com/2220-9964/8/12/559.

[55] Harunor Rashid, Iman Ridda, Catherine King, Matthew Begun, Hatice Tekin, James G. Wood, and Robert Booy. 2015. Evidence compendium and advice on social distancing and other related measures for response to an influenza pandemic. Paediat. Respir. Rev. 16, 2 (2015), 119-126. Retrieved from https://www.ncbi.nlm.nih.gov/pubmed/24630149.

[56] Timothy C. Reluga. 2010. Game theory of social distancing in response to an epidemic. PLoS Comput. Biol. 6, 5 (2010). Retrieved from https://www.ncbi.nlm.nih.gov/pmc/articles/PMC2877723/.

[57] Simon Rogers. 2013. John Snow's data journalism: The cholera map that changed the world. The Guardian (15 Mar. 2013). Retrieved from https://www.theguardian.com/news/datablog/2013/mar/15/john-snow-cholera-map.

[58] Dirk Rothenbücher, Jamy Li, David Sirkin, Brian Mok, and Wendy Ju. 2016. Ghost driver: A field study investigating the interaction between pedestrians and driverless vehicles. In Proceedings of the 25th IEEE International Symposium on Robot and Human Interactive Communication (RO-MAN'16). 795-802. DOI : https://doi.org/10.1109/ROMAN.2016.7745210

[59] Madelyn Sanfilippo, Yan Shvartzshnaider, Irwin Reyes, Helen Nissenbaum, and Serge Egelman. 2020. Disaster privacy/privacy disaster. 7. Assoc. Inf. Sci. Technol. (Feb. 2020). Retrieved from https://papers.ssrn.com/sol3/papers.cfm?abstract_id=3427562.

[60] Stefan Seer, Norbert Brändle, and Carlo Ratti. 2014. Kinects and human kinetics: A new approach for studying pedestrian behavior. Transport. Res. Part C: Emerg. Technol. 48 (2014), 212-228. Retrieved from https://www.sciencedirect.com/science/article/pii/ S0968090X14002289.

[61] Rob Semmens, Nikolas Martelaro, Pushyami Kaveti, Simon Stent, and Wendy Ju. 2019. Is now a good time? An empirical study of vehicle-driver communication timing. In Proceedings of the CHI Conference on Human Factors in Computing Systems. 1-12.

[62] Leonardo Setti, Fabrizio Passarini, Gianluigi De Gennaro, Pierluigi Barbieri, Maria Grazia Perrone, Massimo Borelli, Jolanda Palmisani, Alessia Di Gilio, Prisco Piscitelli, and Alessandro Miani. 2020. Airborne Transmission Route of COVID-19: Why 2 Meters/6 Feet of Inter-personal Distance Could Not Be Enough. Retrieved from https:/www.ncbi.nlm.nih.gov/pmc/articles/PMC7215485/.

[63] Meghendra Singh, Prasenjit Sarkhel, Gloria J. Kang, Achla Marathe, Kevin Boyle, Pamela Murray-Tuite, Kaja M. Abbas, and Samarth Swarup. 2019. Impact of demographic disparities in social distancing and vaccination on influenza epidemics in urban and rural regions of the United States. BMC Infect. Dis. 19, 1 (2019), 221. Retrieved from https://bmcinfectdis.biomedcentral.com/articles/10.1186/s12879019-3703-2.

[64] Christopher Slobogin. 2002. Public privacy: Camera surveillance of public places and the right to anonymity. Miss. Law f. 72 (2002), 213. Retrieved from https://ir.vanderbilt.edu/bitstream/handle/1803/5752/Public\%20Privacy.pdf?sequence=1\&isAllowed=y.

[65] John Snow. 1855. On the Mode of Communication of Cholera. John Churchill.

[66] Steven T. Stoddard, Brett M. Forshey, Amy C. Morrison, Valerie A. Paz-Soldan, Gonzalo M. Vazquez-Prokopec, Helvio Astete, Robert C. Reiner, Stalin Vilcarromero, John P. Elder, Eric S. Halsey et al. 2013. House-to-house human movement drives dengue virus transmission. Proc. Nat. Acad. Sci. 110, 3 (2013), 994-999. Retrieved from https:/www.pnas.org/content/pnas/110/3/994.full.pdf.

[67] Phillip Stroud, Sara Del Valle, Stephen Sydoriak, Jane Riese, and Susan Mniszewski. 2007. Spatial dynamics of pandemic influenza in a massive artificial society. F. Artif. Societ. Soc. Simul. 10, 4 (2007), 9. Retrieved from http://jasss.soc.surrey.ac.uk/10/4/9.html.

[68] Michele Tizzoni, Paolo Bajardi, Adeline Decuyper, Guillaume Kon Kam King, Christian M. Schneider, Vincent Blondel, Zbigniew Smoreda, Marta C. González, and Vittoria Colizza. 2014. On the use of human mobility proxies for modeling epidemics. PLoS Comput. Biol. 10, 7 (2014). Retrieved from https://www.ncbi.nlm.nih.gov/pmc/articles/PMC4091706/pdf/pcbi.1003716.pdf.

[69] Gonzalo M. Vazquez-Prokopec, Donal Bisanzio, Steven T. Stoddard, Valerie Paz-Soldan, Amy C. Morrison, John P. Elder, Jhon RamirezParedes, Eric S. Halsey, Tadeusz J. Kochel, Thomas W. Scott et al. 2013. Using GPS technology to quantify human mobility, dynamic contacts and infectious disease dynamics in a resource-poor urban environment. PloS One 8, 4 (2013). Retrieved from https://www. ncbi.nlm.nih.gov/pmc/articles/PMC3620113/.

[70] John Villasenor. 2011. Recording Everything: Digital Storage as an Enabler of Authoritarian Governments. Center for Technology Innovation at Brookings. Retrieved from https://www.brookings.edu/research/recording-everything-digital-storage-as-an-enabler-ofauthoritarian-governments/.

[71] W. F. Wells. 1934. On air-borne infection: Study II. droplets and droplet nuclei.Amer. F. Epidem. 20, 3 (1934), 611-618.

[72] Amy Wesolowski, Caroline O. Buckee, Kenth Engø-Monsen, and Charlotte Jessica Eland Metcalf. 2016. Connecting mobility to infectious diseases: The promise and limits of mobile phone data. F. Infect. Dis. 214, suppl_4 (2016), S414-S420. Retrieved from https://academic.oup.com/jid/article-abstract/214/suppl_4/S414/2527905.

[73] A. Wilder-Smith and D. O. Freedman. 2020. Isolation, quarantine, social distancing and community containment: Pivotal role for oldstyle public health measures in the novel coronavirus (2019-nCoV) outbreak. f. Trav. Med. 27, 2 (02 2020). DOI : https://doi.org/10.1093/ $\mathrm{jtm} / \mathrm{taaa} 020$

[74] Elizabeth Williamson and Kristin Hussey. 2020. Party zero: How a Soirée in Connecticut became a "Super Spreader." New York Times (23 Mar. 2020). Retrieved from https://www.nytimes.com/2020/03/23/us/coronavirus-westport-connecticut-party-zero.html.

[75] Mary E. Wilson and Lin H. Chen. 2020. Travellers give wings to novel coronavirus (2019-nCoV). F. Trav. Med.icine 27, 2 (2 2020). Retrieved from https://academic.oup.com/jtm/article/27/2/taaa015/5721275.

Digital Government: Research and Practice, Vol. 1, No. 4, Article 32. Publication date: October 2020. 
[76] Zhibin Xiao, Yang Wang, Kun Fu, and Fan Wu. 2017. Identifying different transportation modes from trajectory data using tree-based ensemble classifiers. ISPRS Int. f. Geo-inf. 6, 2 (2017), 57.

[77] Shi Zhao, Peihua Cao, Daozhou Gao, Zian Zhuang, Marc K. C. Chong, Yongli Cai, Jinjun Ran, Kai Wang, Lin Yang, Daihai He et al. 2020. Epidemic growth and reproduction number for the novel coronavirus disease (COVID-19) outbreak on the Diamond Princess cruise ship from January 20 to February 19, 2020: A preliminary data-driven analysis. Retrieved from https://www.ijidonline.com/article/ S1201-9712(20)30091-6/fulltext.

Received July 2019; revised August 2020; accepted August 2020

Digital Government: Research and Practice, Vol. 1, No. 4, Article 32. Publication date: October 2020. 